\title{
Statistics of a parallel Poynting vector in the auroral zone as a function of altitude using Polar EFI and MFE data and Astrid-2 EMMA data
}

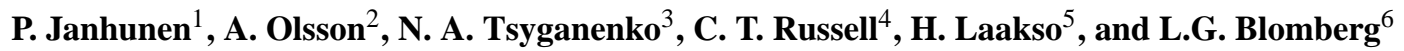 \\ ${ }^{1}$ Finnish Meteorological Institute, Geophysical Research, Helsinki, Finland \\ ${ }^{2}$ Unaffiliated, previously at Swedish Institute of Space Physics, Uppsala, Sweden \\ ${ }^{3}$ Universities Space Research Association, Code 695.1, NASA Goddard Space Flight Centre, Greenbelt, MD 20771, USA \\ ${ }^{4}$ Institute of Geophysics and Planetary Physics, UCLA, Los Angeles, CA 90024-1567, USA \\ ${ }^{5}$ ESTEC, Space Science Department, Noordwijk, The Netherlands \\ ${ }^{6}$ Alfvén Laboratory, Royal Institute of Technology, Stockholm, Sweden
}

Received: 18 October 2004 - Revised: 22 April 2005 - Accepted: 10 May 2005 - Published: 28 July 2005

\begin{abstract}
We study the wave-related (AC) and static (DC) parallel Poynting vector (Poynting energy flux) as a function of altitude in auroral field lines using Polar EFI and MFE data. The study is statistical and contains 5 years of data in the altitude range $5000-30000 \mathrm{~km}$. We verify the low altitude part of the results by comparison with earlier Astrid-2 EMMA Poynting vector statistics at $1000 \mathrm{~km}$ altitude. The EMMA data are also used to statistically compensate the Polar results for the missing zonal electric field component. We compare the Poynting vector with previous statistical DMSP satellite data concerning the electron precipitation power. We find that the AC Poynting vector (Alfvénwave related Poynting vector) is statistically not sufficient to power auroral electron precipitation, although it may, for $K_{p}>2$, power $25-50 \%$ of it. The statistical AC Poynting vector also has a stepwise transition at $R=4 R_{E}$, so that its amplitude increases with increasing altitude. We suggest that this corresponds to Alfvén waves being in Landau resonance with electrons, so that wave-induced electron acceleration takes place at this altitude range, which was earlier named the Alfvén Resonosphere (ARS). The DC Poynting vector is $\sim 3$ times larger than electron precipitation and corresponds mainly to ionospheric Joule heating. In the morning sector (02:00-06:00 MLT) we find that the DC Poynting vector has a nontrivial altitude profile such that it decreases by a factor of $\sim 2$ when moving upward from 3 to $4 R_{E}$ radial distance. In other nightside MLT sectors the altitude profile is more uniform. The morning sector nontrivial altitude profile may be due to divergence of the perpendicular Poynting vector field at $R=3-4 R_{E}$.
\end{abstract}

Correspondence to: P. Janhunen

(pekka.janhunen@fmi.fi)
Keywords. Magnetospheric physics (Auroral phenomena; Magnetosphere-ionosphere interactions) - Space plasma physics (Wave-particle interactions)

\section{Introduction}

Energy is dissipated in the auroral ionosphere via charged particle (mainly electron) precipitation and resistive Joule heating. Statistically, the level of the particle precipitation is rather well known (Hardy et al., 1987, 1989). There are also several statistical satellite studies of the Joule heating (Foster et al., 1983; Gary et al., 1995; Olsson et al., 2004a) that are in quite good agreement with each other, despite the different types of instrumentation used (Olsson et al., 2004a).

The common picture of auroral energy budget is that most energy arrives from the magnetosphere as electromagnetic energy flux (Poynting vector) and dissipates in the ionosphere as Joule heating. A smaller amount of energy arrives in the form of energetic particles (mainly electrons), causing auroral particle precipitation. In addition, some part of the electromagnetic energy flux is transferred to electron energy flux (auroral acceleration) before reaching the ionosphere.

Observationally, how the precipitation and Joule heating power arrive from the magnetosphere has not yet been mapped in quantitative detail. For example, it is not known how the different particle and electromagnetic parts of the total energy flux vector vary with altitude and what their absolute and relative magnitudes are in different conditions. Recently, it was shown to be consistent with Polar data that in some events, it is energetically possible for Alfvén waves observed at $R=4-5 R_{E}$ radial distance to explain the simultaneously observed intense substorm-related electron 
precipitation at the satellite footpoint (Wygant et al., 2000, 2002). Later it was shown by Keiling et al. (2003) that statistically, $\approx 1 / 3$ of auroral electron precipitation power can be explained by time-varying (alternating current, AC) Poynting flux at $4-5 R_{E}$ radial distance.

This paper presents a statistical study on the parallel component of the Poynting vector and attempts to answer how the electromagnetic energy flux arrives from the magnetosphere along field lines to the ionospheric plane. The use of the Poynting vector to discuss ionosphere-magnetosphere energy balance was introduced by Sugiura (1984) and the mathematical aspects were refined by Kelley et al. (1991). Somewhat inaccurately, the parallel Poynting vector is in the literature often simply called the "Poynting flux". Unless otherwise stated, by "parallel Poynting vector" or just "Poynting vector" we mean the parallel component of the Poynting vector. The papers present both AC and DC (direct current) parallel Poynting vector profiles as a function of radial distance $R$ in different MLT sectors and other geophysical conditions. The perpendicular Poynting vector components and the electron and ion energy fluxes are outside the scope of the study. Their contribution is not necessarily small, as our conclusions will show.

\section{Instrumentation}

We use Polar Electric Field Investigation (EFI, Harvey et al., 1995) and Magnetic Field Experiment (MFE, Russell et al., 1995) instruments to compute the parallel Poynting vector. The EFI time resolution is always at least 20 samples per second and the MFE time resolution is 8 samples per second. The time interval April 1996 to July 2001 is used, selecting from each orbit only those data that map to the nominal nightside auroral oval (18:00-06:00 MLT, 65-74 ILAT).

Only the spin-plane components of the electric field are used in this study, which effectively means that only the meridional (north-south) component of the electric field is used. This component is usually larger than the zonal (eastwest) component because auroral features are normally eastwest aligned. To approximately calibrate the Polar statistical result with respect to the missing electric field component, we use a recent work where the low-altitude $(1000 \mathrm{~km})$ parallel Poynting vector was studied using Astrid-2 data (Blomberg et al., 2004; Olsson et al., 2004a). The spin plane of Astrid-2 is nominally perpendicular to the EarthSun line, which makes it possible in many cases to accurately resolve the 3-D electric field using the $\boldsymbol{E} \times \boldsymbol{B}=0$ assumption (Ivchenko et al., 2001; Olsson et al., 2004a). We reprocessed the Astrid-2 data set by setting the zonal electric field artificially to zero. The result was that the parallel Poynting vector was then underestimated by a factor of $\sim 1.5$. On physical grounds, this correction factor should fall in between 1 and 2 , with 1 corresponding to completely meridional electric fields and 2 corresponding to zonal and meridional electric field amplitudes being equally large, so the Astrid-2 implied correction factor of 1.5 is reasonable because it falls clearly in between the two extremes 1 and 2. We assume that the same correction factor applies to all altitudes, i.e. we multiply all statistical Polar Poynting vector results by 1.5 . Notice that this correction factor implicitly includes both the physical effects (zonal wave electric field also contributing to the Poynting vector), as well as the effects due to the Polar spin plane being not exactly meridionally aligned.

\section{Computation of parallel Poynting vector}

The parallel component of the Poynting vector is

$S_{\|}=\left(\frac{\boldsymbol{B}_{0}}{B_{0}}\right) \cdot \frac{1}{\mu_{0}} \boldsymbol{E} \times\left(\boldsymbol{B}-\boldsymbol{B}_{0}\right)$,

where $\boldsymbol{E}$ and $\boldsymbol{B}$ are the measured electric and magnetic fields and $\boldsymbol{B}_{0}$ is a curl-free background magnetic field model. The background field should contain the effect of external current systems (e.g. Chapman-Ferraro current and tail current) as accurately as possible, but not those flowing inside the volume at the boundary of which the Poynting vector is computed, i.e. the background $\boldsymbol{B}_{0}$ should be curl-free in the volume enclosed by the orbit of the satellite.

We consider both static (DC) and time-varying (AC) parallel Poynting vector. In the DC Poynting vector case we define $\boldsymbol{B}_{0}$ to be the T01s model (Tsyganenko et al., 2003), from which the Birkeland current (field-aligned current, FAC) and nonsymmetric ring current parts are removed to obtain a curl-free background field in an Earth-centred sphere of radius $6 R_{E}$ (the highest radial distance of Polar used in this study is $6 R_{E}$ ). We call the resulting background model T01s_NOFAC. The model is driven by the observed solar wind/IMF parameters and the $D_{s t}(\mathrm{SYM})$ index (5-min resolution).

In the AC Poynting vector case we define $\boldsymbol{B}_{0}$ as a lowpass, filtered version of the measured $\boldsymbol{B}$. The filter cutoff in time domain is obtained from the mean ILAT speed of Polar (averaged over the auroral crossing in question) and a desired spatial cutoff in ILAT domain (expressed in kilometres in ionospheric plane). For the ILAT cutoffs we use values of $100 \mathrm{~km}, 200 \mathrm{~km}$ and $500 \mathrm{~km}$. For example, at $R=4 R_{E}$, Polar's ILAT speed is $\sim 300 \mathrm{~m} / \mathrm{s}$ (Janhunen et al., 2004a, , Fig. 2), giving time-domain cutoffs of $5.5 \mathrm{~min}, 11 \mathrm{~min}$ and 28 min, respectively. We also tried time-domain filters (e.g. using 2-min altitude-independent cutoff), but since the results do not bring any qualitatively new features, they are not shown.

In Fig. 1 we show an example event from 10 May 1997 when Polar was at 4-5 $R_{E}$ radial distance close to $22 \mathrm{mag}$ netic local time (MLT). The raw $\boldsymbol{E}$ and $\boldsymbol{B}$ fields (panels (a-f)) have been averaged to $1 \mathrm{~s}$ resolution before plotting, although the full resolution is used in the Poynting vector computation. The parallel AC Poynting vector (panels $(g, h, i)$ ) clearly depends on the spatial cutoff employed: the maximum value is $\sim 2 \mathrm{~mW} \mathrm{~m}^{-2}$ for the $100 \mathrm{~km}$ cutoff and $\sim 8 \mathrm{~mW} \mathrm{~m}^{-2}$ for the $500 \mathrm{~km}$ cutoff. The DC parallel Poynting vector (panel (j)) reaches more than $15 \mathrm{~mW} \mathrm{~m}^{-2}$. Both $\mathrm{AC}$ and DC parallel 
Poynting vectors are predominantly downward. In this case, there is an upward Region-1 FAC at the poleward edge of the oval (68-69 ILAT) and a downward Region-2 FAC at the equatorward edge (around 67 ILAT). The closing Pedersen current flowing in between the current sheets involves Joule heating, which comes from the downward Poynting vector.

In this paper the parallel Poynting vector is always mapped to the ionospheric plane using flux tube scaling.

\section{Results}

\subsection{Radial distance}

Figure 2 is a summary plot of the Poynting vector statistics for the overall nightside auroral oval; the following plots (Figs. 3-5) in the paper have the same structure, but they will show limited statistics using some further criteria. Figure 2 shows the orbital coverage (top), the AC parallel Poynting vector for spatial cutoffs 100,200 and $500 \mathrm{~km}$, and the DC parallel Poynting vector (bottom). As we explained above, all Polar Poynting vectors are multiplied by 1.5 to compensate statistically for the missing zonal electric field component. Figure 2 also shows the statistical low-altitude parallel Poynting vector from a recent Astrid-2 study (Olsson et al., 2004a), as well as the statistical electron precipitation from the model of Hardy et al. (1987), which is based on DMSP satellite measurements. The latter is shown in order to obtain a better understanding of the auroral acceleration and Poynting to electron energy conversion processes. Both Astrid-2 and DMSP data are taken at $\sim 1.1 R_{E}$ radial distance, i.e. well below the acceleration region. The altitude coverage of these satellites is very narrow and therefore their statistics are presented on both sides of $R=1$ (Earth's surface), in order to improve their visibility; DMSP statistics appear between $R=0.7-1$ and Astrid-2 between $R=1-1.3$. The Epstein function coefficients given in Table 4 of Hardy et al. (1987) were used to construct MLT and ILAT dependent expressions, which were then averaged over the MLT and ILAT range in question. The Hardy et al. (1987) coefficients are given separately for each integer $K_{p}$; to obtain the results for the $K_{p}$ ranges $K p \leq 2$ and $K_{p}>2$, we used the observed $K_{p}$ occurrence frequencies during 1996-2001.

From Fig. 2 we draw four main conclusions: (1) The DC parallel Poynting vector from Polar agrees (after multiplication by 1.5 , see above) with the Astrid- 2 result and is clearly larger (especially for $K_{p}>2$ ) than the electron precipitation power (panel (e)); (2) the AC parallel Poynting vector is, on the other hand, clearly smaller than the electron precipitation, including the largest $(500 \mathrm{~km})$ spatial cutoff (panels (b,c,d)); (3) for $K_{p}>2$, there is a rather clear step in the AC Poynting vector at $4 R_{E}$ radial distance (panels (b, c, d)); (4) there is a decreasing trend in the DC Poynting vector (panel (e)) as a function of increasing radial distance.

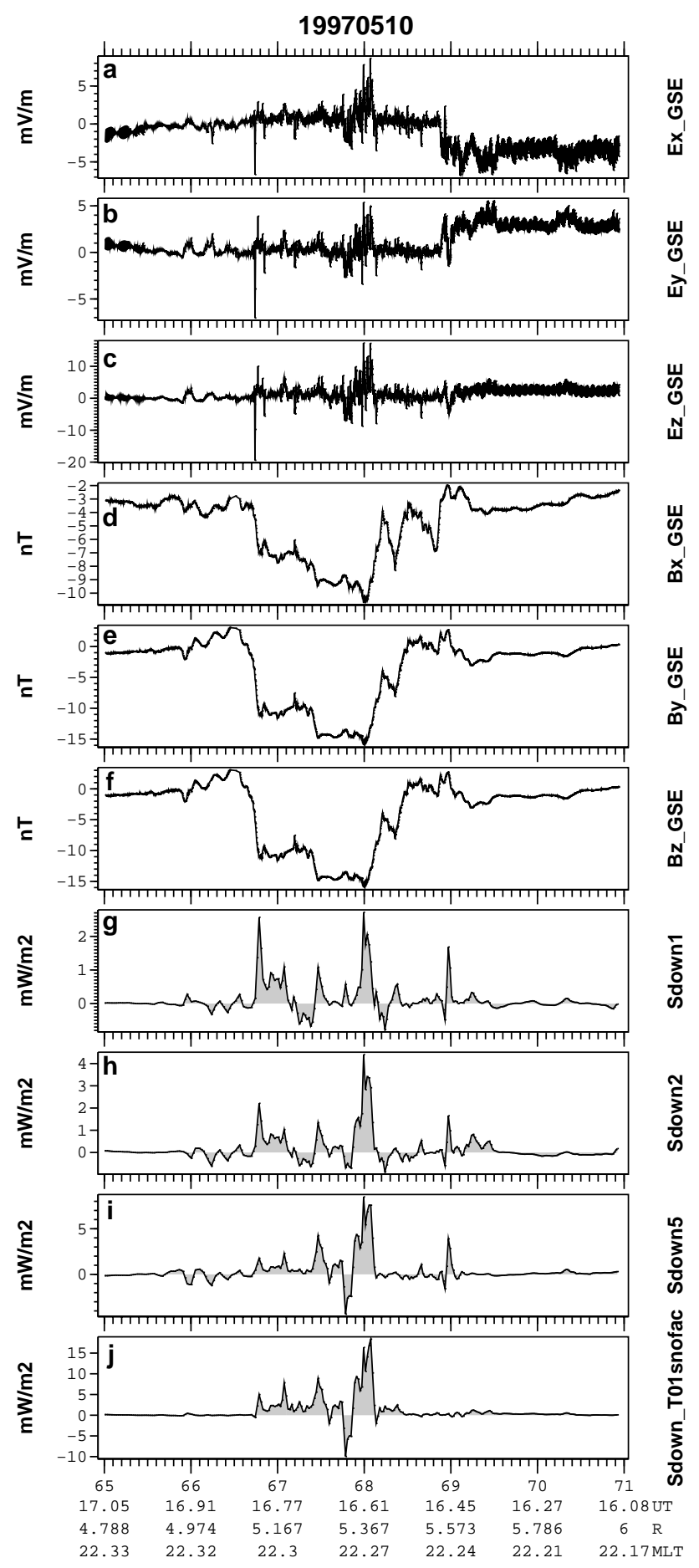

Fig. 1. Example event 10 May 1997. Cartesian GSE components of measured spin-plane EFI electric field $(\mathbf{a}-\mathbf{c})$, GSE components of MFE magnetic field with T01s_NOFAC background subtracted (d-f), Fourier-filtered downward AC Poynting vector for ionospheric scales less than $100 \mathrm{~km}, 200 \mathrm{~km}, 500 \mathrm{~km}((\mathrm{~g}-\mathbf{i})$, respectively) and total downward DC Poynting vector with T01s_NOFAC model subtraction $(\mathbf{j})$. 

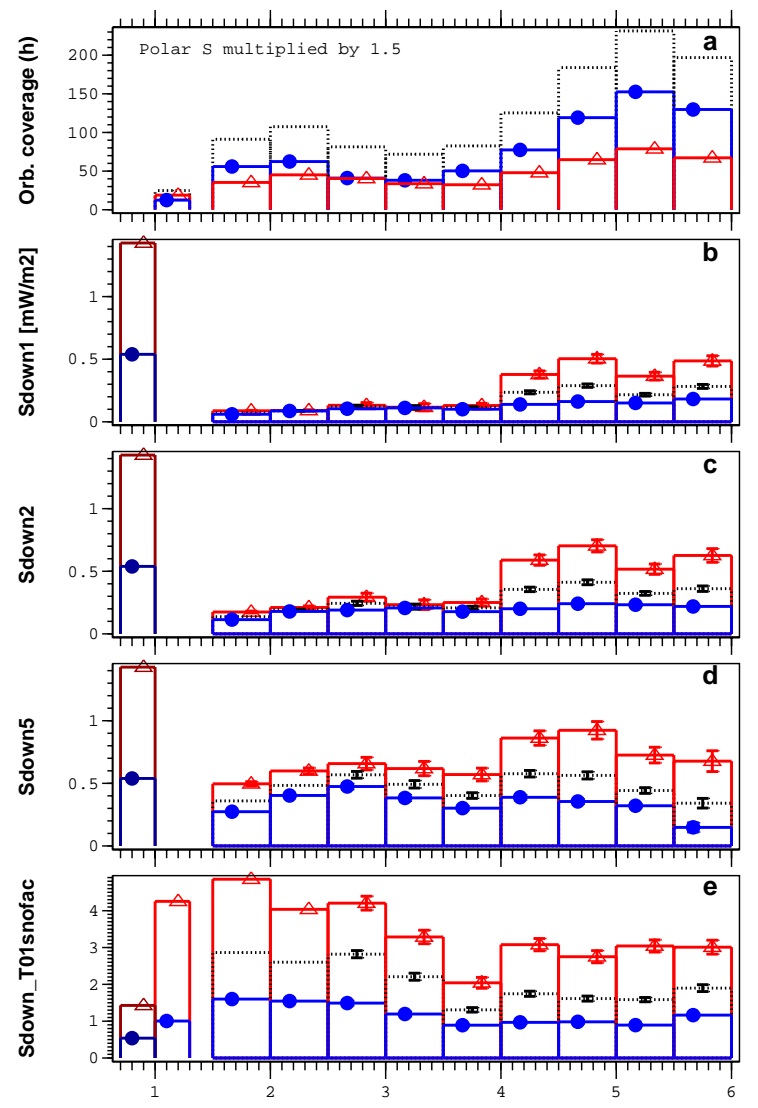

$R / R \_E$

Fig. 2. Statistics of parallel Poynting vectors for the overall nightside auroral oval. Polar orbital coverage as a function of radial (geocentric) distance $R$ in hours with $K_{p} \leq 2$ shown as blue solid dots, $K_{p}>2$ as red triangles and each $K_{p}$ with a black dotted line (a), downward AC Poynting vector mapped to ionospheric plane (in $\mathrm{mW} \mathrm{m}^{-2}$ ) for ionospheric scales less than $100 \mathrm{~km}, 200$ and $500 \mathrm{~km}$ (b-d), respectively, and total downward DC Poynting vector with T01_NOFAC model field subtraction (e). In panels (b-e), dark-blue and reddish bar at $R=0.7-1$ is electron precipitation power per area from the Hardy et al. (1987) model for low $K_{p}(\leq 2)$ and high $K_{p}(>2)$, respectively. In panel (e), blue and red bar at $R=1-1.3$ is DC Poynting vector from Astrid-2 satellite $(1000 \mathrm{~km}$ altitude) from Olsson et al. (2004a) and in panel (a), the corresponding bar shows the orbital coverage of Astrid-2. To compensate for the fact that only the spin-plane components of Polar electric field are used, all measured Polar Poynting vectors are multiplied by 1.5 (see text for motivation).

\subsection{Error sources of Poynting vector measurement}

The accuracy of the DC Poynting vector depends on the accuracy of the magnetic field measurement, the accuracy of the electric field measurement and on the accuracy of the background model field.

To derive an upper limit for the magnetic field measurement error, we selected all low-altitude $(B>5000 \mathrm{nT})$ Polar MFE data where $K_{p} \leq 1$, and determined the daily median directional difference between the measured field and the T01s model field. The value obtained represents the sum of the pointing error and the deviation between nature and the T01s
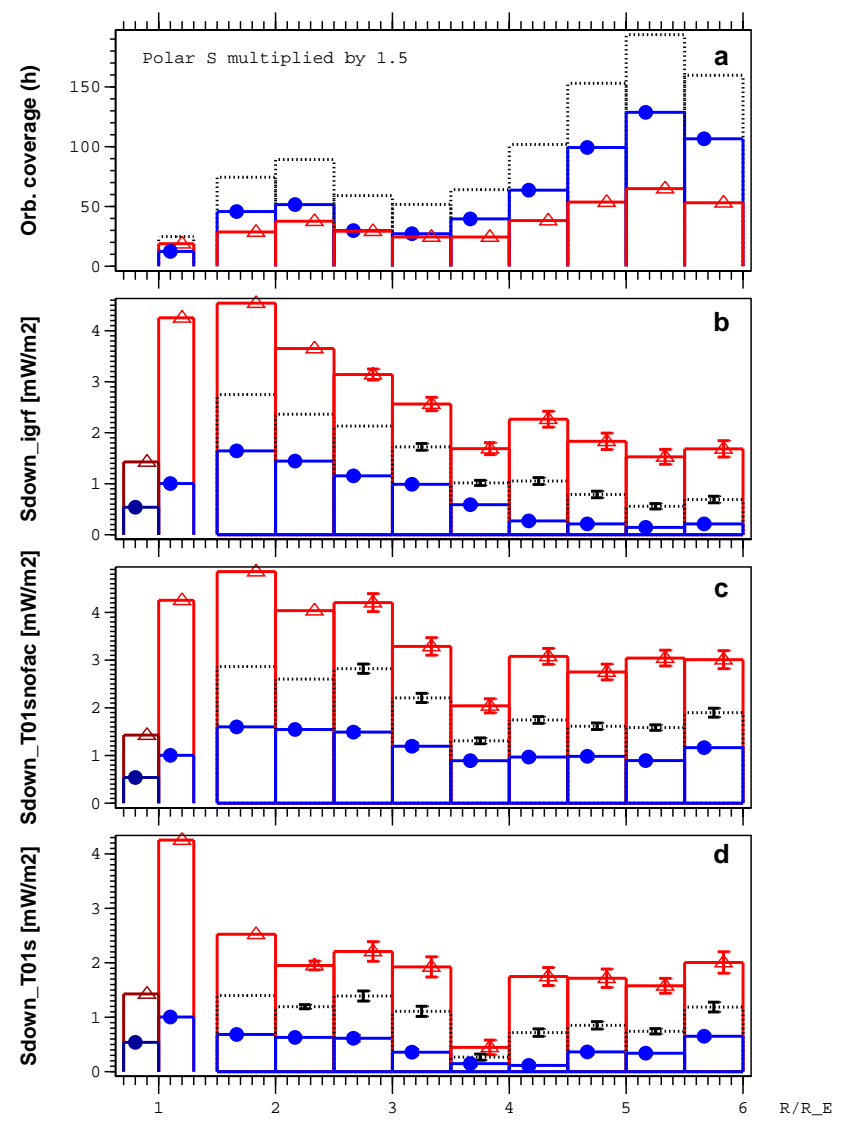

Fig. 3. Distribution of DC Poynting vector against distance for three background magnetic field models. Panels from top to bottom: orbital coverage (a), IGRF backgrond (b), T01s_NOFAC background (the one used in other figures) (c) and T01s background (d). Otherwise format similar to Fig. 2.

model. The latter is expected to be minimised when $K_{p}$ is small and the altitude is low. The smallest routinely occurring daily values obtained in this way are about $0.15^{\circ}$, which we take as an upper limit for possible pointing inaccuracy. The $0.15^{\circ}$ pointing error would translate to a $26-n T$ spurious perpendicular $B$-field, in the worst case, where the background field is the largest concerning Polar (10000 nT). With a reasonable ionospheric electric field of $30 \mathrm{mV} / \mathrm{m}$ the associated maximum spurious Poynting vector is $1.4 \mathrm{~mW} \mathrm{~m}^{-2}$ when mapped to the ionosphere. This is small compared to the typically occuring peak values (e.g. $10-15 \mathrm{~mW} \mathrm{~m}^{-2}$ in the example of Fig. 1), from which the Poynting flux obtains most of its contribution. We emphasise that this is an upper limit for the possible error due to pointing inaccuracy, not an estimate of the likely level of pointing inaccuracy which might be much smaller.

One usually estimates that the Polar double-probe electric field measurement accuracy is about $1 \mathrm{mV} / \mathrm{m}$. Taking again $30 \mathrm{mV} / \mathrm{m}$ as representative of a typical ionospheric field where significant Poynting flux may occur, the fluxtube scaled field at $R=6 R_{E}$ is $2 \mathrm{mV} / \mathrm{m}$. Thus, at the highest altitudes covered the Polar $E$-field inaccuracy may start to become an issue. 

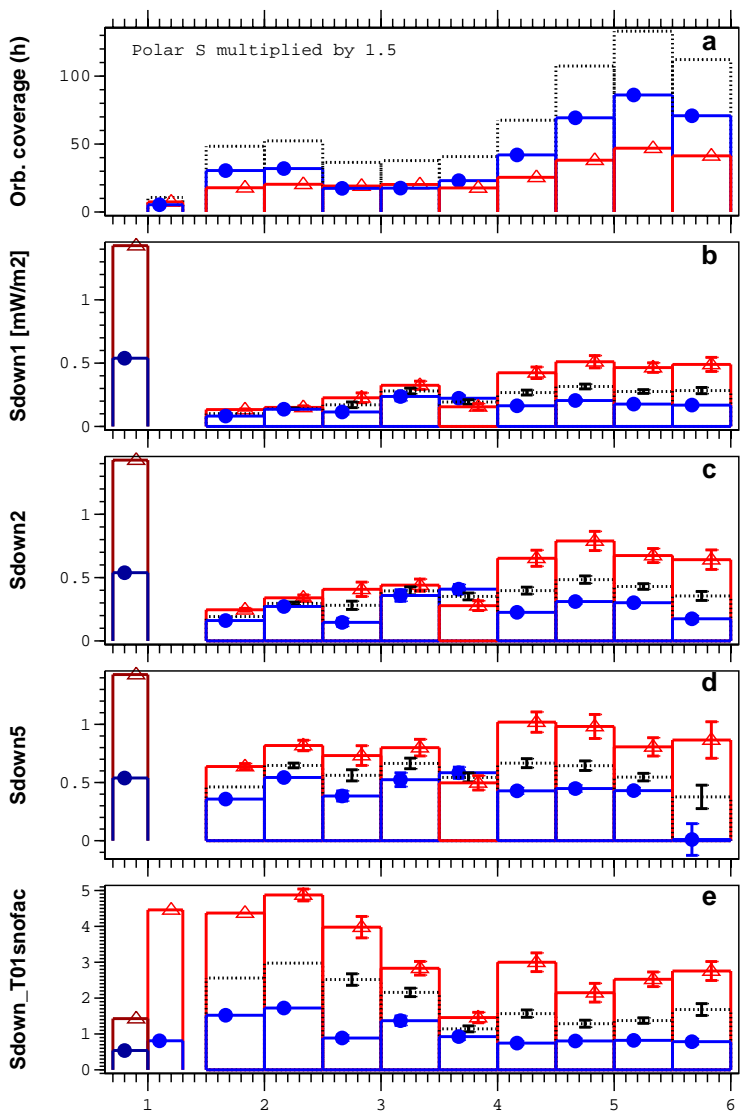
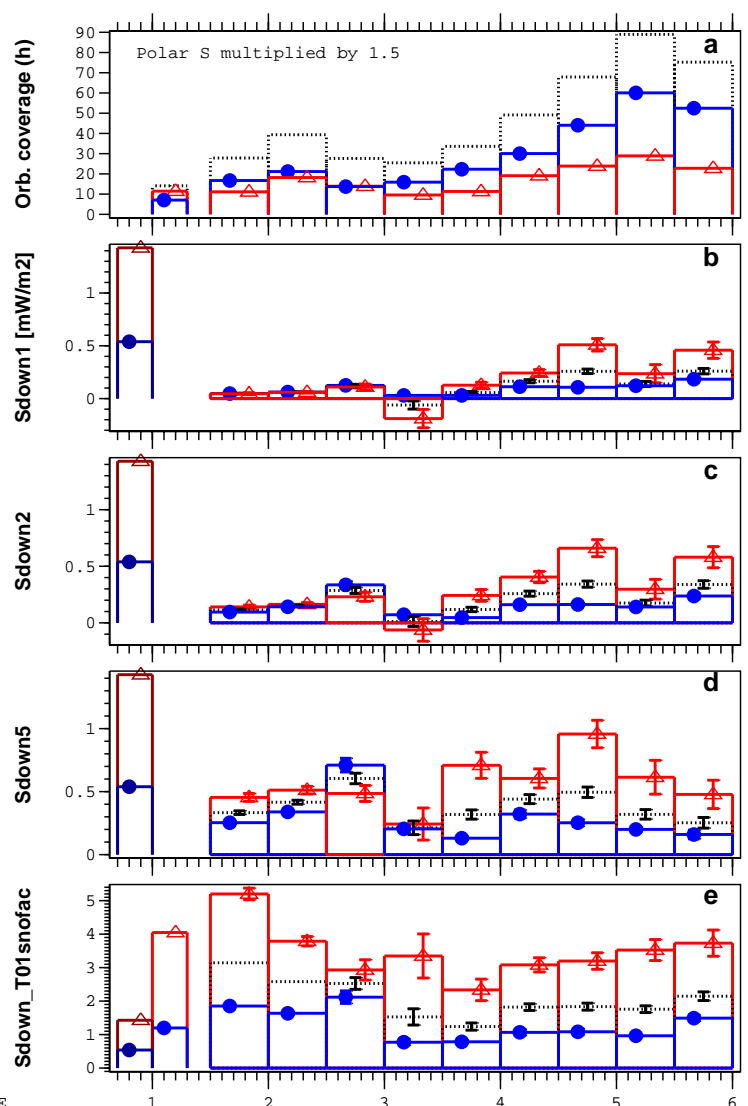

$R / R$

Fig. 4. Same as Fig. 2, but decomposed into cases where Polar ionospheric footpoint is in darkness (left) or illuminated by the Sun (right).

In Astrid-2 we estimate that the pointing accuracy is the dominant source of error and that it is about $0.1^{\circ}$. Because Astrid-2 altitude is lower $(1000 \mathrm{~km})$, the resulting spurious $B$-field is somewhat larger $(70 \mathrm{nT})$ than for Polar, but still of the same order of magnitude.

To address the background model issue, in Fig. 3 we show the DC Poynting vector as in Fig. 2, panel (e), but now for different versions of the background magnetic field model: the IGRF internal field model only (panel (b)), the T01s_NOFAC (panel (c)) and T01s, i.e. the T01s without FAC removal (panel (d)). The Astrid-2 Poynting vector and Hardy et al. (1987) electron precipitation results are also shown in each panel for comparison. We see that for radial distance up to $3.5 R_{E}$ or so, the IGRF and T01s_NOFAC model results agree fairly well. This is not surprising, since at low altitude the IGRF part of T01s_NOFAC dominates anyway. At higher altitudes, the results differ progressively more and more, which is natural, taking into account that the IGRF does not model, for example, the nightside stretching of the magnetosphere at all. The T01s without FAC removal (panel (d)) gives a clearly smaller DC Poynting vector than the other models. This is also natural, since if one subtracts the magnetic effects of the FACs, the Poynting vector associated with those FACs is then not counted.
In a large statistical study such as this, errors like pointing inaccuracy that are random and independent of the measured quantity are statistically reduced by a factor $1 / \sqrt{N}$, where $N$ is the number of independent measurements. In this paper $N$ (if taken to be the number of auroral crossings contributing to each data bin) is at least of the order of 100, whence the errors due to pointing inaccuracies and $E$-field measurement are reduced by a large factor (at least 10 ), making them probably quite insignificant. We believe that the dominant source of error in the statistical results of this paper comes from the fact that the intensity of auroral events varies in large limits, so that the most intense events may not be negligible when the average power is considered. Perhaps the safest way to have an idea of the magnitude of this effect is to simply compare adjacent radial bins in Figs. 2-5: to the extent that they display consistent behaviour, the results can be trusted.

\subsection{Solar illumination and MLT}

In Fig. 4 we show the measurements of Fig. 2 in the same style, but the data are decomposed into two groups for darkness and sunlit ionospheric footpoint conditions. Interestingly there is no systematic difference, other than in the sunlit case the AC Poynting vector altitude profile exhibits more irregular variations, including some net upward Poynting vectors in some radial bins. 

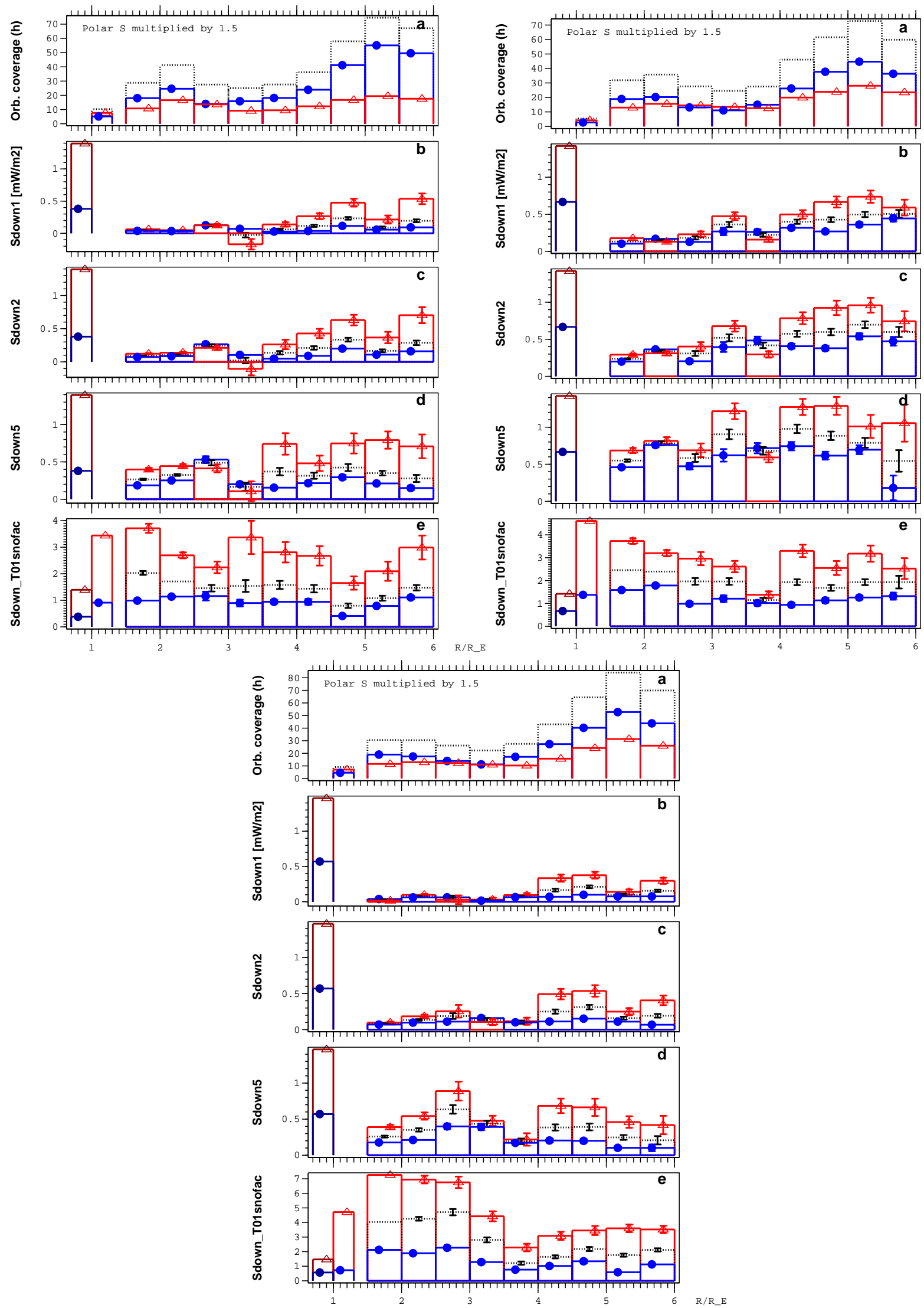

Fig. 5. Same as Fig. 2, but decomposed into three nightside MLT sectors, 18-22, 22-02 and 02-06. 
Figure 5 is as Fig. 2, but now the data are decomposed into three nightside MLT sectors (18:00-22:00, 22:00-02:00 and 02:00-06:00). The AC Poynting vectors are the largest in the midnight MLT sector. We now see that the decreasing trend in the DC Poynting vector (property 4 in Sect. 4.1) is mainly due to the morning sector, where the Poynting vector is almost constant below $R=3 R_{E}$ and above $R=4 R_{E}$, but a significant step-like change occurs in between. Also in the midnight sector, a slight decreasing trend as a function of $R$ is seen.

Regarding the amount of statistical scatter, the error bars displayed in the plots correspond to $1 / \sqrt{N}$ error, where $N$ is the number of auroral crossings contributing to each bin (missing error bar means that the error bar would be too small to be visible). In cases where the solar wind has temporal correlations comparable to Polar's orbital period $(18 \mathrm{~h})$, the resulting error bars may be underestimates because then two consecutive orbits are not fully independent. The fact that adjacent bins sometimes fluctuate more than the error bars (e.g. bin 3-3.5 $R_{E}$ for high $K_{p}$ in panel (d) of right subplot of Fig. 4) insinuates that this may be the case to some extent in the data set under study. It is important in this type of study to pay attention to both the magnitude of the error bars and the apparent bin-to-bin fluctuation level.

\subsection{MLT-ILAT dependence}

Figure 6 shows the MLT-ILAT distribution of the AC and DC Poynting vectors, averaged below $2.5 R_{E}$ radial distance (upper subplots) and averaged over 4-5.5 $R_{E}$ (lower subplots). In each case, the left subplot shows the low $K_{p}$ result $\left(K_{p} \leq 2\right)$ and the right subplot shows the high $K_{p}$ result $\left(K_{p}>2\right)$. The top panel in each subplot is the orbital coverage, thereafter follow the three $\mathrm{AC}$ panels and the bottom panel is the DC Poynting vector, as usual. The gray scale is fixed to $0-1 \mathrm{~mW} \mathrm{~m}^{-2}$ in the AC Poynting vector panels and to $0-7 \mathrm{~mW} \mathrm{~m}^{-2}$ in the DC panels. The low $K_{p}$ side shows that both the AC and DC Poynting vectors follow an MLTILAT pattern which is reminiscent of the average auroral oval, in the same way as, for example, electron anisotropies have been found to follow the oval (Janhunen et al., 2004c). A preference of large AC Poynting vectors that appear in the midnight MLT sector is also clearly visible. On the high $K_{p}$ side, similar trends are seen, except that there is naturally more statistical scatter and also the average oval resides at lower ILAT than for low $K_{p}$, which is also not surprising. A step in the altitude profile of the AC Poynting vector for high $K_{p}$ seen in Fig. 2 is also visible here: the high $K_{p}$ AC Poynting vectors at high altitude are clearly larger than at low altitude.

\section{Summary and Discussion}

One purpose of this paper is to study statistically whether the AC parallel Poynting vector (wave-related Poynting vector) alone can power auroral electron precipitation as suggested earlier (Wygant et al., 2000, 2002). We have therefore studied how the AC Poynting vector varies with altitude using Polar data in the altitude range $5000-30000 \mathrm{~km}$. To be able to compare with the energy flux needed to power the auroral electron precipitation we include statistics from Hardy et al. (1987), using DMSP electron data (altitude about $800 \mathrm{~km}$ ).

Furthermore, to obtain a better understanding for the energy transfer processes in the auroral zone, we also study the altitude profile of the DC component of the Poynting vector, in addition to the $\mathrm{AC}$ one. Recall that the definitions of "DC" and "AC" Poynting vectors differ by how the subtracted background magnetic field is defined: in the DC case it is a T01s model field and in the AC case it is a low-pass filtered version of the measured field. As an independent verification we use recent DC Poynting vector statistical results from Astrid-2 EMMA instrument at $1000 \mathrm{~km}$ altitude (Olsson et al., 2004a). The same Astrid-2 data set is also used to calibrate the Polar statistics with respect to the unmeasured zonal electric field component (notice that the latter is logically independent of the former). The DC values of the Poynting vectors are to be compared with the abovementioned electron energy fluxes from the DMSP satellite at about $800 \mathrm{~km}$. Below we summarize our findings.

1. The average energy input to the nightside (18:0006:00 MLT) auroral zone (65-74 ILAT) is 1.5 and $5.5 \mathrm{~mW} \mathrm{~m}^{-2}$ for low $(\leq 2)$ and high $(>2) K_{p}$, respectively. About $30 \%$ of the energy input is particle precipitation and $70 \%$ is Joule heating. These numbers are obtained by combining the present results and/or our earlier Astrid-2 results (Olsson et al., 2004a) with the electron precipitation data of Hardy et al. (1987).

2. At least below $R=6 R_{E}$, the main energy input arrives as a parallel (DC) Poynting vector from the magnetosphere. However, in the morning sector the DC Poynting vector decreases by a factor of $\sim 2$ when moving from $R=3 R_{E}$ to $R=4 R_{E}$. A slight decreasing trend is also seen in the midnight sector, while in the evening sector the DC flux is almost independent of altitude. While this result is somewhat sensitive to the background model, we believe that the morningside decreasing behaviour is real.

3. Statistically, the AC Poynting vector alone cannot power auroral electron precipitation. Even for high $K_{p}$, the highest AC Poynting vector values are only half of the observed electron energy flux (Fig. 2, panels (b-d)). The part that goes into electron energisation is likely to be even smaller (see discussion on ARS below).

4. The waves carrying significant Poynting vectors are probably substorm-related, since the AC Poynting vector is dominant for high $K_{p}$ and in the midnight sector (Fig. 5, panels (b-d)). It is most likely that these waves are Alfvén waves. 


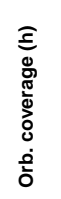
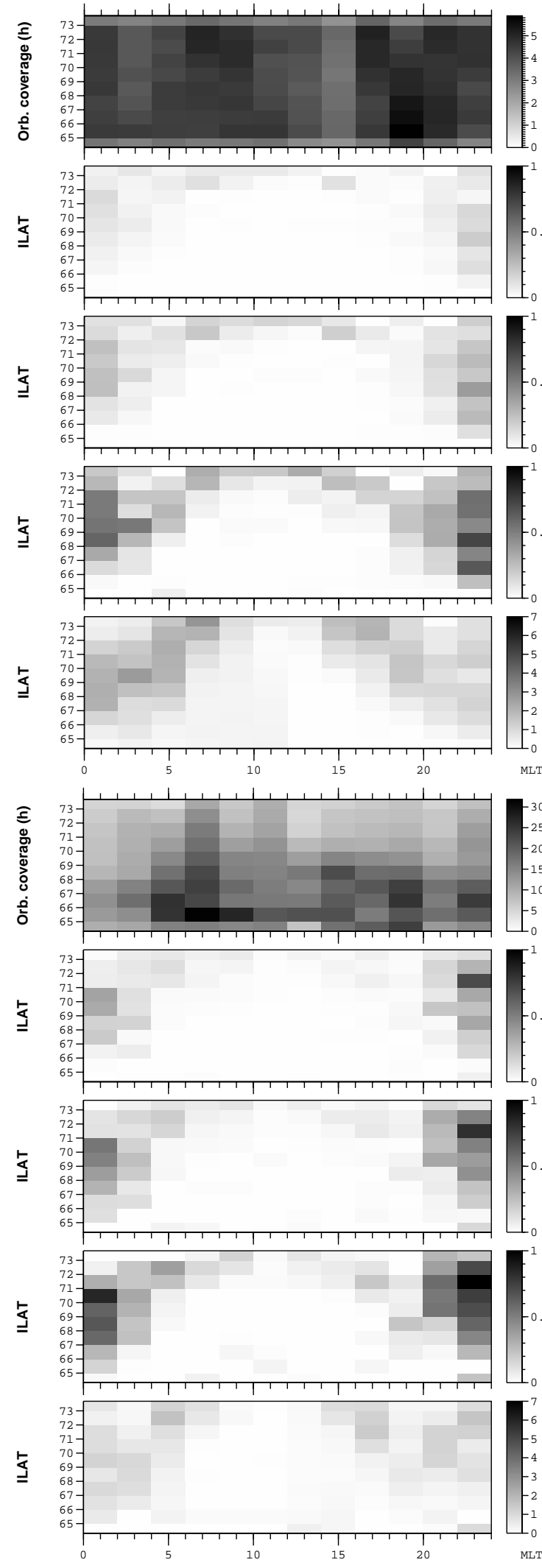
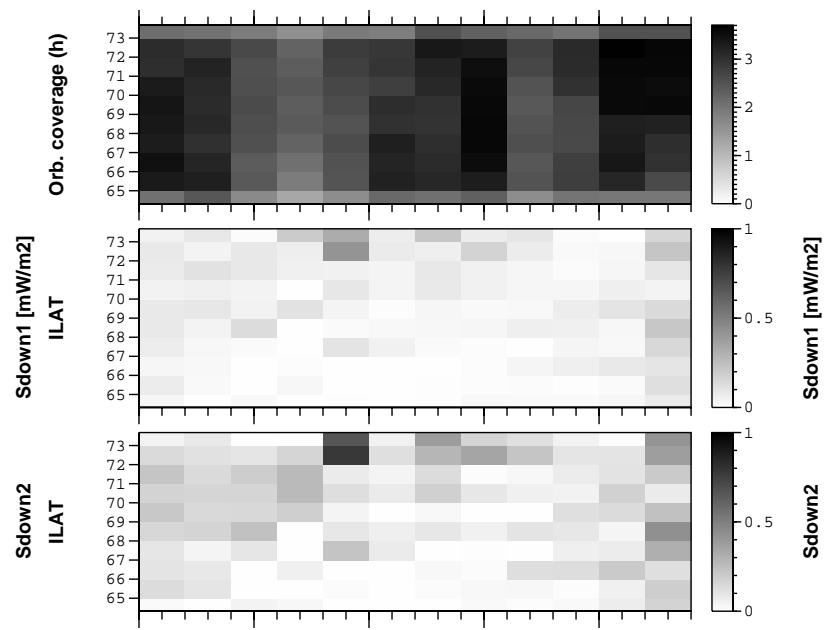

蒙
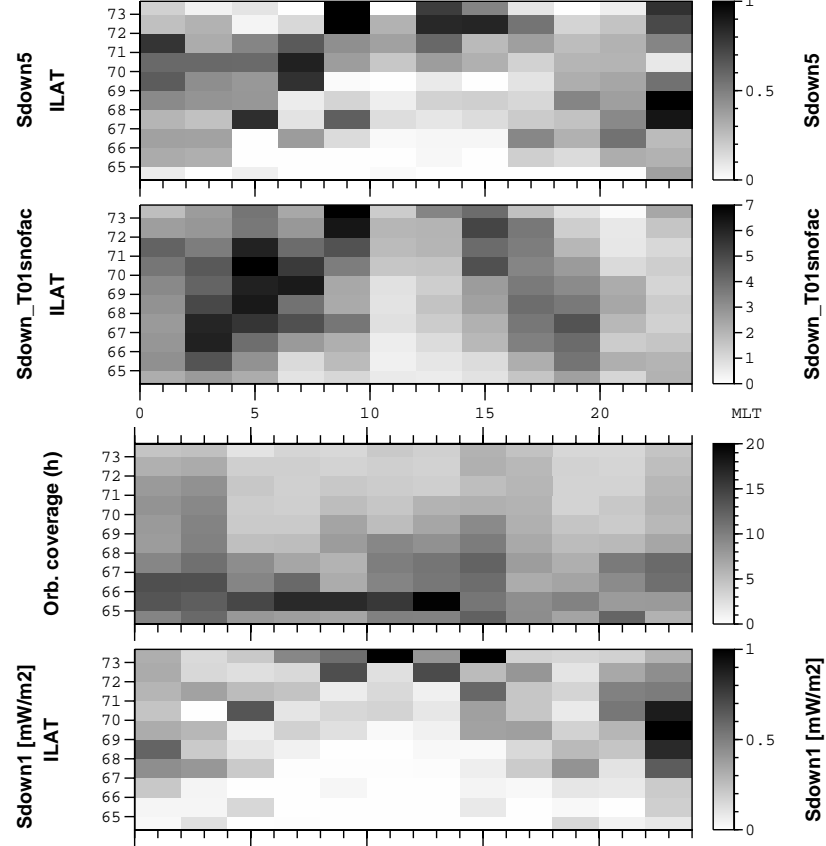

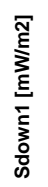
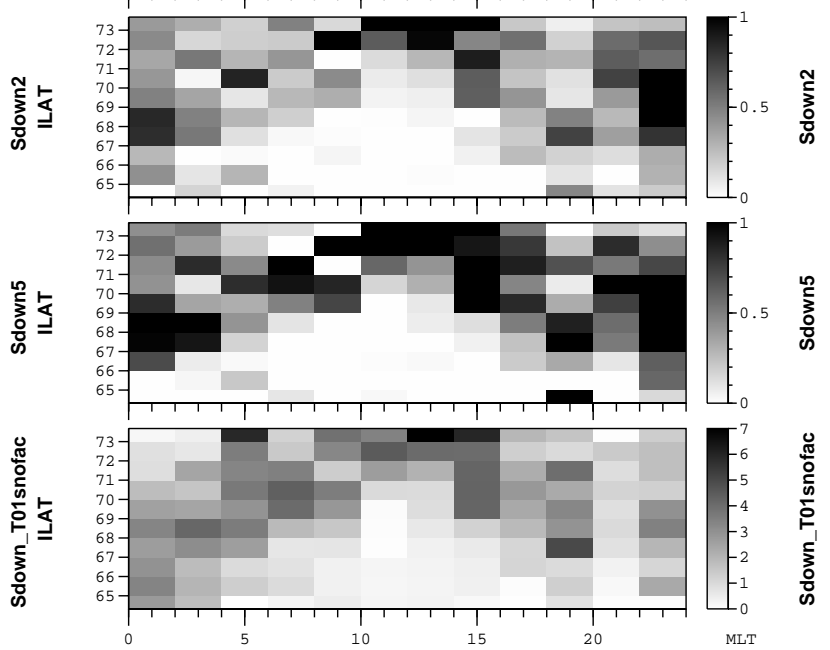

Fig. 6. Polar downward Poynting vector as a function of MLT and ILAT, low altitude $\left(1-2.5 R_{E}\right)$ in top row and high altitude (4-5.5 $\left.R_{E}\right)$ in bottom row, low $K_{p}(\leq 2)$ on the left and high $K_{p}(>2)$ on the right. In each subplot, top panel is orbital coverage in hours, then follow AC Poynting vectors at ionospheric scale sizes smaller than $100 \mathrm{~km}, 200 \mathrm{~km}$ and $500 \mathrm{~km}$. The bottom panel is the total DC Poynting vector with T01s_NOFAC model field subtraction. 

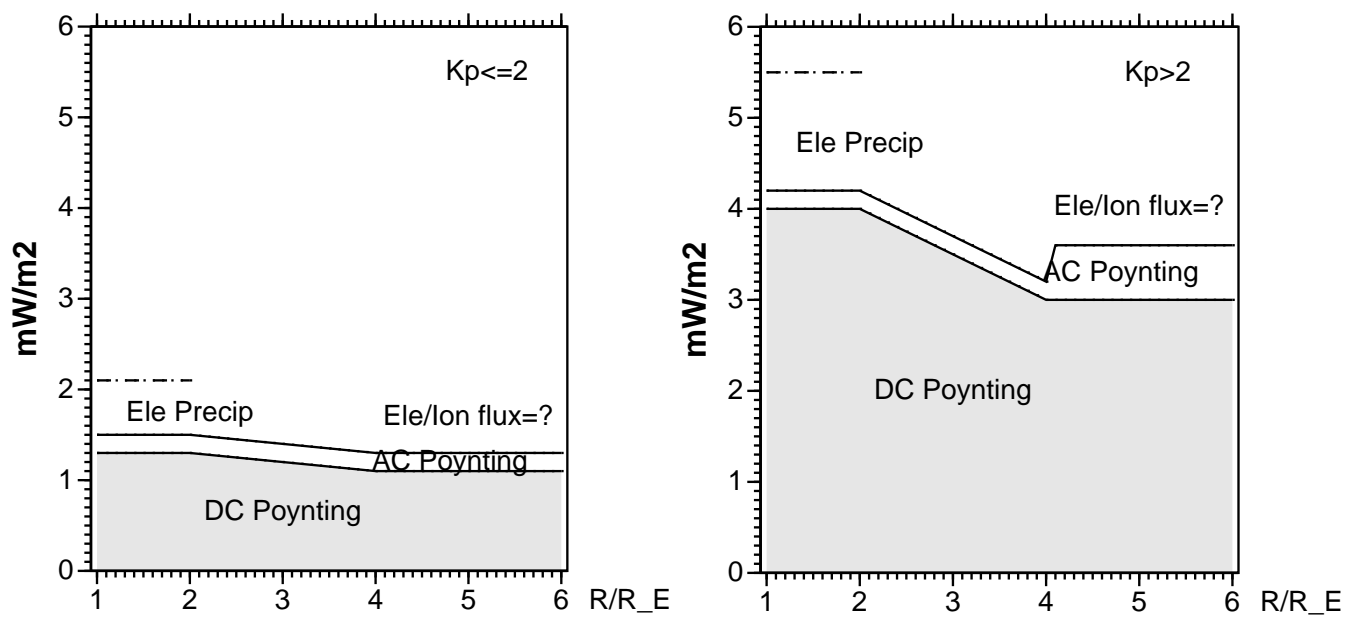

Fig. 7. Schematic description of power per footpoint area of DC Poynting vector, AC Poynting vector (less than $200 \mathrm{~km}$ ionospheric scale size) and auroral electron precipitation power. The DC Poynting vector is larger than the electron precipitation, which is in turn larger than AC Poynting vector.

5. For high $K_{p}$, the wave-related Poynting vector abruptly decreases at $4 R_{E}$ when moving downward (Fig. 2, panels (b) and (c), see also (d)), suggesting that incoming Alfvénic wave power is partly transformed to particle energy at this altitude. Notice that there is no evidence that the part of the wave power extending down to the lowest radial bin would be converted to particle energy: it can also be converted into time-varying ionospheric Joule heating. The AC Poynting vector "step" at $4 R_{E}$ corresponds to about $1 / 3$ of the electron precipitation.

6. For low $K_{p}$, the AC Poynting vector is low and independent of the altitude (the exact value depends on the spatial cutoff chosen).

7. The AC and DC Poynting vectors follow clearly the auroral zone (Fig. 6). The wave Poynting vector prefers the midnight auroral zone.

These findings raise some natural questions to which we now try to give some plausible answers:

1. Into what form of energy does the high- $K_{p}$ AC Poynting vector transform to at $4 R_{E}$ ? Recently, the concept of an "Alfvén Resonosphere" (ARS) was coined to explain a local increase in the occurrence frequency of density cavities at $4 R_{E}$ (Janhunen et al., 2004b). The new AC Poynting vector results of this paper are in complete agreement with and directly support the proposed idea that incoming transient Alfvén waves experience a Landau resonance with electrons at the ARS altitude (Olsson et al., 2004b; Janhunen et al., 2004b; Wygant et al., 2002). Since the Alfvén speed is directly proportional to the magnetic field which varies as $R^{-3}$, the ARS is a rather well-defined layer rather than an extended region.
2. If only $1 / 3$ of the auroral electron precipitation can be powered by Alfvénic electron energisation at ARS during $K_{p}>2$ and less for $K_{p} \leq 2$, then where does the remaining dominant part come from? This study does not give a definite answer. The possibilities are the DC parallel Poynting vector and hot electron or ion energy flux components. To our knowledge, no statistical attempts have been made to estimate or to constrain these energy flux components.

3. Why does the parallel DC Poynting vector decrease between 3 and $4 R_{E}$ radial distance in the morning sector (see Fig. 5)? It is possible that perpendicular Poynting vector divergence feeds the parallel one in this altitude range if the total divergence of the Poynting vector is nonzero. The altitude range where this happens $\left(R=3-4 R_{E}\right)$ is near the statistical upper boundary of auroral cavities (Janhunen et al., 2002); perhaps significant perpendicular currents flow only in regions where there is enough plasma and plasma pressure gradient to cancel the Lorentz force caused by the current. The perpendicular Poynting vector might be associated with a closure current feeding the Region-2 current system. We know of no natural explanation why the evening sector behaves differently in this respect, however.

To the end, Fig. 7 summarises our results for the magnitudes of the AC and DC parallel Poynting vector and electron precipitation. The DC Poynting vector dominates other forms of energy transfer and goes predominantly to ionospheric Joule heating. The AC Poynting vector is a small perturbation in comparison; for low $K_{p}$ the AC flux is independent of altitude, thus showing no signs of energy transfer to particles, but for high $K_{p}$ there is a transition at $R=4 R_{E}$, which is consistent with the ARS idea. We remark that this transition occurs close to $R=3-4 R_{E}$ radial range, where in 
the morning (and to a lesser extent the midnight) MLT sector the DC Poynting vector decreases with increasing distance. Finally, the question as to what powers the auroral precipitation is still open after this study: while the large magnitude of the DC Poynting vector could leave room for it, other possibilities, such as hot ion and electron energy fluxes, and a perpendicular Poynting vector, should also be addressed in the future.

Acknowledgements. The work of AO was supported by the Swedish Research Council and that of PJ by the Academy of Finland. The work at UCLA was supported by NASA grant NAG5 11324. The authors thank Andris Vaivads for useful comments.

The Editor in chief thanks a referee for his/her help in evaluating this paper.

\section{References}

Blomberg, L. G., Marklund, G. T., Lindqvist, P.-A., Primdahl, F., Brauer, P., Bylander, L., Cumnock, J. A., Eriksson, S., Ivchenko, N., Karlsson, T., Kullen, A., Merayo, J. M. G., Pedersen, E. B., Petersen, J. R.: EMMA - the Electric and Magnetic Monitor of the Aurora on Astrid-2, Ann. Geophys., 22, 115-123, 2004,

SRef-ID: 1432-0576/ag/2004-22-115.

Foster, J. C., St.-Maurice, J.-P., and Abreu, V. J.: Joule heating at high latitudes, J. Geophys. Res., 88, 4885-4896, 1983.

Gary, J. B., Heelis, R. A. and Thayer, J. P.: Summary of fieldaligned Poynting flux observations from DE 2, Geophys. Res. Lett., 22, 1861-1864, 1995.

Hardy, D. A., Gussenhoven, M. S., Raistrick, R., and McNeil, W. J.: Statistical and functional representations of the pattern of auroral energy flux, number flux, and conductivity, J. Geophys. Res., 92, 12 275-12 294, 1987.

Hardy, D. A., Gussenhoven, M. S. and Brautigam, D.: A statistical model of auroral ion precipitation, J. Geophys. Res., 94, 370392, 1989.

Harvey, P., Mozer, F. S., Pankow, D., Wygant, J., Maynard, N. C., Singer, H., Sullivan, W., Anderson, P. B., Pfaff, R., Aggson, T., Pedersen, A., Fälthammar, C. G. and Tanskanen, P.: The electric field instrument on the Polar satellite, Space Sci. Rev., 71, 583596, 1995.

Ivchenko, N., Facciolo, L., Lindqvist, P.-A., Kekkonen, P., and Holback, B.: Disturbance of plasma environment in the vicinity of the Astrid-2 microsatellite, Ann. Geophys., 19, 655-666, 2001, SRef-ID: 1432-0576/ag/2001-19-655.

Janhunen, P., Olsson, A., and Laakso, H.: The occurrence frequency of auroral potential structures and electric fields as a function of altitude using Polar/EFI data, Ann. Geophys., 22, 1233-1250, 2004a, SRef-ID: 1432-0576/ag/2004-22-1233.
Janhunen, P., Olsson, A., Hanasz, J., Russell, C. T., Laakso, H. and Samson, J. C.: Different Alfvén wave acceleration processes of electrons in substorms at $4-5 R_{E}$ and 2-3 $R_{E}$ radial distance, Ann. Geophys., 22, 2213-2227, 2004b,

SRef-ID: 1432-0576/ag/2004-22-2213.

Janhunen, P., Olsson, A., and Laakso, H.: Middle-energy electron anisotropies in the auroral region, Ann. Geophys., 22, 237-249, 2004c,

SRef-ID: 1432-0576/ag/2004-22-237.

Janhunen, P., Olsson, A., and Laakso, H.: Altitude dependence of plasma density in the auroral zone, Ann. Geophys., 20, 17431750, 2002,

SRef-ID: 1432-0576/ag/2002-20-1743.

Keiling, A., Wygant, J. R., Cattell, C. A., Mozer, R. S., and Russell, C. T.: The global morphology of wave Poynting flux: powering the aurora, Science, 299, 383-386, 2003.

Kelley, M. C., Knudsen, D. J., and Vickrey, J. F.: Poyntingflux measurements on a satellite: A diagnostic tool for space research, J. Geophys. Res., 96, 201-207, 1991.

Olsson, A., Janhunen, P., Karlsson, T., Ivchenko, N., and Blomberg, L. G.: Statistics of Joule heating in the auroral zone and polar cap using Astrid-2 satellite Poynting flux, Ann. Geophys., 22, 41334142, 2004a,

SRef-ID: 1432-0576/ag/2004-22-4133.

Olsson, A., Janhunen, P., Hanasz, J., Mogilevsky, M. M., Perraut, S. and Menietti, J. D.: Observational study of generation mechanism of substorm-associated low-frequency AKR emissions, Ann. Geophys., 22, 3571-3582, 2004b,

SRef-ID: 1432-0576/ag/2004-22-3571.

Sugiura, M.: A fundamental magnetosphere-ionosphere coupling mode involving field-aligned currents as deduced from DE-2 observations, Geophys. Res. Lett., 11, 877-880, 1984.

Russell, C. T., Snare, R. C., Means, J. D., Pierce, D., Dearborn, D., Larson, M., Barr, G., and Le, G.: The GGS/Polar Magnetic Fields Investigation, Space Sci. Rev., 71, 563-582, 1995.

Tsyganenko, N. A., Singer, H. J. and Kasper, J. C.: Stormtime distortion of the inner magnetosphere: How severe can it get?, J. Geophys. Res., 108, A5, SMP 18-1, CiteID 1209, doi:10.1029/2002JA009808, 2003.

Wygant, J. R., Keiling, A., Cattell, C. A., et al.: Polar spacecraft based comparisons of intense electric fields and Poynting flux near and within the plasma sheet-tail lobe boundary to UVI images: an energy source for the aurora, J. Geophys. Res., 105, 18 675-18 692, 2000.

Wygant, J. R., Keiling, A., Cattell, C. A., et al.: Evidence for kinetic Alfvén waves and parallel electron energization at 4-6 $R_{E}$ altitudes in the plasma sheet boundary layer, J. Geophys. Res., 107, A8, doi:10.1029/2001JA900113, 2002. 